\title{
FIXED POINT THEOREMS FOR CONDENSING MULTIFUNCTIONS
}

\section{J. HIMMELBERG, ${ }^{1}$ J. R. PORTER ${ }^{2}$ AND F. S. VAN VLECK ${ }^{3}$}

1. Introduction. In [9] B. N. Sadovskii introduced the concept of condensing function for functions defined on subsets of a Banach space and obtained thereby a generalization of the Schauder fixed point theorem [10]. In this note we extend Sadovskii's definition to apply to multifunctions defined on subsets of locally convex spaces. We then obtain a single fixed point theorem which contains the theorems of Sadovskii [9], Tychonoff [11], and Fan [4], and part of a theorem of F. E. Browder [3].

In $\$ 2$ we define and develop the condensing concept and prove our main theorem-a fixed point theorem for condensing multifunctions with closed graph, convex values, complete and convex domain, and bounded range. In $\$ 3$ we give another approach to the same problem by defining condensing in terms of the hyperspace uniformity. It is shown that this approach reduces to that of $\$ 2$, if we restrict our attention to compact valued multifunctions. In $\$ 4$ we replace the closed graph hypothesis (which is implied by upper semicontinuity under our conditions) by lower semicontinuity.

Most of the terminology is standard. But to be specific, recall that a multifunction $F: X \rightarrow Y$ is a function which assigns to each point $x$ of $X$ a nonempty subset $F(x)$ of $Y$. Equivalently, $F$ is a subset of $X \times Y$ whose domain $\left(=F^{-1}(Y)\right)$ is $X$. Depending on one's point of view the set $\{(x, y) \mid y \in F(x)\}$ is called either the graph of $F$, or, simply, $F$. If $\Omega \subset X$, then $F(\Omega)=\bigcup\{F(x) \mid x \in \Omega\}$. A submultifunction $G: \Omega \rightarrow Y$ of $F$ is a multifunction from $\Omega$ to $Y$ such that $G(x) \subset F(x)$ for all $x \in \Omega$.

A fixed point of a multifunction $F$ is any point $x$ such that $x \in F(x)$. Clearly any fixed point of a submultifunction of $F$ is a fixed point of $F$.

2. Condensing multifunctions. Let $E$ be a locally convex linear topological space (briefly, locally convex space) and let $Q$ be a basis of neighborhoods of 0 composed of convex sets. If $\Omega \subset E$, define $Q(\Omega)$ to be the collection of all $B \in ß$ such that $S+B \supset \Omega$ for some precompact subset $S$ of $E$. The set $Q(\Omega)$ is a measure of the precompactness of $\Omega$.

Received by the editors January 10, 1969.

1 Supported in part by National Science Foundation Grant GP-6490.

2 Supported in part by University of Kansas Research Grant No. 3416-5038.

' Supported in part by National Science Foundation Grant GY-4422. 
I.e., the larger $Q(\Omega)$ is, the more nearly is $\Omega$ precompact. In fact we have:

Proposition $1 . \Omega$ is precompact iff $Q(\Omega)=B$.

Proof. The "only if" part is trivial. On the other hand, suppose $Q(\Omega)=B$ and let $B \in B$. Choose $V \in B$ such that $V+V \subset B$ and let $S$ be a precompact set such that $S+V \supset \Omega$. Then there exists a finite set $F$ such that $F+V \supset S$. Hence $F+B \supset F+V+V \supset S+V \supset \Omega$. It follows that $\Omega$ is precompact.

Proposition 2. Let $\operatorname{co} \Omega$ be the convex hull of $\Omega$. Then $Q(\operatorname{co} \Omega)=Q(\Omega)$.

Proof. It is clearly sufficient to prove $Q($ co $\Omega) \supset Q(\Omega)$. So let $B \in Q(\Omega)$, and let $S$ be a precompact set such that $S+B \supset \Omega$. Then co $S$ is also precompact. Moreover, $\operatorname{co} S+B \supset \operatorname{co} \Omega$, since $\operatorname{co} S+B$ is convex and co $S+B \supset \Omega$. Hence $B \in Q(\cos \Omega)$.

Definition. Let $T$ be a subset of the locally convex space $E$. A multifunction $F: T \rightarrow E$ is condensing iff for some choice of basis $B$ of convex neighborhoods of 0 , we have $Q(F(\Omega)) \supsetneqq Q(\Omega)$ for every bounded but not precompact subset $\Omega$ of $T$.

The sense of the above definition is that condensing multifunctions take bounded but not precompact sets to sets which are more nearly precompact. It is not difficult to prove that if $E$ is a Banach space and if $B$ is the collection of spherical neighborhoods of 0 , then this definition of condensing is implied by Sadovskii's.

If $E$ is a locally convex space and $T \subset E$, then it is evident that a multifunction $F$ is condensing when either (i) $T$ is compact, or (ii) $F$ takes bounded sets to precompact sets. It follows that Theorem 1 below contains the fixed point theorems of Fan [4], Schauder [10], and Tychonoff [11]. If $F$ is a function, then (ii) plus continuity is the definition of complete continuity of $F$. It is thus tempting to define complete continuity of a multifunction $F$ to mean that $F$ satisfies (ii) and some continuity condition. However, Browder [3] has already defined this concept in a manner which is more general in one way and more restrictive in another. His definition allows him to obtain an improved version of Corollary 2 below (with, however, the restriction that $T=E$ ).

Lemma 1. Let $X$ be a topological space, and let $F: X \rightarrow X$ be a multifunction with closed graph. If there is a nonempty subset $A$ of $X$ such that $\bar{A}$ is compact and $F(A) \subset A$, then there exists a nonempty, closed, and compact subset $K$ of $X$ such that $F(K) \supset K$. 
Proof. Let $G=F \cap(\bar{A} \times \bar{A})$. Then $G$ is closed and compact, and $G^{-1}(\bar{A})$ is a closed subset of $\bar{A}$ containing $A$. Hence, dom $G$ $=G^{-1}(\bar{A})=\bar{A}$. I.e., $G$ is a multifunction from the compact set $\bar{A}$ to itself. By $[1$, p. 113 , Theorem 8$]$ there is a nonempty, closed, and compact set $K$ such that $G(K)=K$. Clearly, $F(K) \supset K$.

THEOREM 1. Let $T$ be a nonempty complete convex subset of a separated locally convex space $E$, and let $F: T \rightarrow T$ be a condensing multifunction with convex values, closed graph, and bounded range. Then $F$ has a fixed point.

REMARK. Theorem 1 remains true for nonseparated $E$ if we add the assumption that $T$ is closed. No essential change in the proof is necessary. This is so because the same remark applies to Fan's fixed point theorem. To prove this version of Fan's theorem, use the customary replacement of a nonseparated locally convex space by a separated one.

Proof of Theorem 1. By hypothesis, $F(T)$ is bounded. Let $\Omega$ be the closed convex hull of $F(T)$. Then $F(\Omega) \subset \Omega$, and $\Omega$ is bounded, complete, and convex. We will prove that $F$ has a fixed point in $\Omega$ by an argument which parallels Sadovskii's.

First apply Lemma 1 to find a nonempty compact subset $K$ of $\Omega$ such that $F(K) \supset K$. To do this we need $A \subset \Omega$ such that $\bar{A}$ is compact and $F(A) \subset A$. Let $x \in \Omega$, and let $A=\{x\} \cup F(x) \cup F^{2}(x) \cup \ldots$ Then $A$ is bounded, and $A=\{x\} \cup F(A)$. Hence, by the condensing property, $A$ is precompact. But $\bar{A} \subset \Omega$ and hence $\bar{A}$ is compact. Trivially $F(A) \subset A$.

Now define a transfinite sequence of convex sets $\Omega_{\alpha}$ by $\Omega_{0}=\Omega$, and

$$
\begin{aligned}
\Omega_{\alpha} & =\operatorname{co} F\left(\Omega_{\alpha-1}\right), \quad \text { if } \alpha \text { is not a limit ordinal, } \\
& =\bigcap\left\{\Omega_{\beta} \mid \beta<\alpha\right\}, \quad \text { if } \alpha \text { is a limit ordinal. }
\end{aligned}
$$

It is easily verified that each of the following assertions is true for all $\alpha$ :

(i) $\Omega_{\alpha}$ is a convex subset of $\Omega$,

(ii) $F\left(\Omega_{\alpha}\right) \subset \Omega_{\alpha}$,

(iii) $K \subset \Omega_{\alpha}$ and hence $\Omega_{\alpha} \neq \varnothing$.

Since the sequence $\left(\Omega_{\alpha}\right)$ is nonincreasing and hence eventually constant, there exists $\gamma$ such that $\operatorname{co} F\left(\Omega_{\gamma}\right)=\Omega_{\gamma+1}=\Omega_{\gamma}$. Then

$$
Q\left(F\left(\Omega_{\gamma}\right)\right)=Q\left(\operatorname{co} F\left(\Omega_{\gamma}\right)\right)=Q\left(\Omega_{\gamma}\right),
$$

so that, by the condensing property, $\Omega_{\gamma}$ is precompact, and $\bar{\Omega}_{\gamma}$ is a compact convex subset of $\Omega$. 
Let $G=F \cap\left(\bar{\Omega}_{\gamma} \times \bar{\Omega}_{\gamma}\right)$. By the argument in the first part of the proof of the lemma, $G$ is a multifunction from $\bar{\Omega}_{\gamma}$ to $\bar{\Omega}_{\gamma}$. Moreover $G$ has convex values and compact graph. It follows easily that $G^{-1}(C)$ is closed for each closed subset $C$ of $\bar{\Omega}_{\gamma}$, i.e., $G$ is upper semicontinuous. By Fan's fixed point theorem, $G$ has a fixed point. This point is also a fixed point of $F$.

REMARK. The following corollaries are partial generalizations of Theorem 3 in Browder [3]. He assumes a stronger complete continuity condition in order to allow a much weaker boundedness condition on $F(T)$ (namely that $F^{n}(T)$ be bounded for some $n \geqq 1$ ).

COROLlary 1. Let $T$ be a nonempty complete convex subset of a separated locally convex space, and let $F: T \rightarrow T$ be a multifunction with bounded range. Also, suppose that, for each bounded subset $\Omega$ of $T$, there exists a condensing submultifunction $G: \Omega \rightarrow T$ of $F$ with closed graph and convex values. Then $F$ has a fixed point.

Proof. Let $\Omega$ be the closed convex hull of $F(T)$. Then $\Omega$ is bounded, convex, and complete. Let $G: \Omega \rightarrow T$ be as in the hypotheses. Then $G(\Omega) \subset F(\Omega) \subset F(T) \subset \Omega$. So $G$ has a fixed point by Theorem 1 .

Corollary 2. Let $T$ be a nonempty complete convex subset of a separated locally convex linear space, and let $F: T \rightarrow T$ be a multifunction with convex values, closed graph, and bounded range. Moreover, suppose that, for each bounded subset $\Omega$ of $T$, there exists a precompact subset $K$ of $T$ such that $F \cap(\Omega \times K)$ is a multifunction from $\Omega$ to $K$. Then $F$ has a fixed point.

Proof. Let $\Omega$ be a bounded set and $K$ a precompact set such that $F \cap(\Omega \times K)$ is a multifunction from $\Omega$ to $K$. Then

$$
G=F \cap(\Omega \times \overline{\operatorname{co}} K): \Omega \rightarrow T
$$

has closed graph and convex values. So apply Corollary 1 .

3. Condensing functions into hyperspaces. Let $E$ be a locally convex linear space and denote by $\mathfrak{C}(E)$ the set of all nonempty closed compact subsets of $E$. Let $B$ be a basis of convex neighborhoods of 0 , and give $\mathfrak{C}(E)$ the uniform structure which has as basis the collection of all sets of the form

$$
\tilde{B}=\{(M, N) \in \mathfrak{e}(E) \times \mathfrak{e}(E) \mid M \subset N+B \text { and } N \subset M+B\},
$$

where $B \in B$. This uniform structure is the subspace structure on $\mathfrak{e}(E)$ inherited from the hyperspace uniform structure on the set $2^{E}$ of nonempty closed subsets of $E$. (The latter uniform structure is 
assumed to be induced by the natural uniform structure on $E$ in the usual way. See $[2$, p. 206] or [6].)

The results of $\$ 2$ suggest that we measure the precompactness of a subset $W$ of $\mathrm{C}(E)$ by the set $Q_{h}(W)$ of all $B \in Q$ such that $\tilde{B}(\mathcal{S}) \supset W$ for some precompact subset $\mathcal{S}$ of $\mathfrak{C}(E)$. As in $\S 2$, $W$ is precompact iff $Q_{h}(W)=B$.

Now let $T \subset E$ and let $F: T \rightarrow E$ be a multifunction with closed compact values. We may regard $F$ as a function $F_{h}: T \rightarrow \mathfrak{C}(E)$. Define $F$ to be $h$-condensing iff there exists a basis $B$ of convex neighborhoods of 0 such that $Q_{h}\left(F_{h}(\Omega)\right) \supsetneqq Q(\Omega)$ for every bounded but not precompact subset $\Omega$ of $T$. An example of such a multifunction is any $F: T \rightarrow E$ which has closed compact values and for which the corresponding function $F_{h}: T \rightarrow \mathfrak{e}(E)$ takes bounded subsets of $E$ to precompact subsets of $\mathfrak{C}(E)$. It develops that $h$-condensing implies condensing. However, we can use $\mathfrak{C}(E)$ to define a condensing concept weaker than $h$-condensing, but sometimes equivalent to condensing in the sense of $\$ 2$.

Let $B$ be a basis of convex neighborhoods of 0 . Then the collection of all sets of the form

$$
\widetilde{B}_{r}=\{(M, N) \in \mathfrak{e}(E) \times \mathfrak{e}(E) \mid N \subset M+B\},
$$

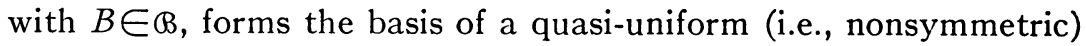
uniform structure on $\mathfrak{C}(E)$. This structure is coarser than the hyperspace uniform structure, since $\widetilde{B} \subset \widetilde{B}_{r}$ for all $B \in ß$. For each subset W of $\mathrm{e}(E)$, define $Q_{r}(W)$ to be the set of all $B \in \Theta$ such that $\widetilde{B}_{r}(\mathcal{S}) \supset W$ for some precompact subset $\mathcal{S}$ of $\mathfrak{C}(E)$. (Here the precompactness of $\mathcal{S}$ is defined in terms of the hyperspace uniform structure.) Then a multifunction $F: T \rightarrow E$ is $r$-condensing iff there exists a basis $B$ of convex neighborhoods of 0 such that $Q_{r}\left(F_{h}(\Omega)\right) \supsetneqq Q(\Omega)$ for every bounded but not precompact subset $\Omega$ of $T$.

Proposition 3. Let $@$ be a basis of convex neighborhoods of 0 , and let $W \subset \mathrm{e}(E)$. Then $Q(\mathrm{U} W) \supset Q_{r}(W) \supset Q_{h}(W)$. Furthermore, if $E$ is complete, then $Q(\cup W)=Q_{r}(W)$.

Proof. Trivially $Q_{r}(W) \supset Q_{h}(W)$. So let $B \in Q_{r}(W)$, and let $s$ be a precompact subset of $\mathfrak{C}(E)$ such that $\tilde{B}_{r}(\mathcal{S}) \supset W$. It is easily shown that $S=\bigcup S$ is precompact. Furthermore $S+B \supset \cup w$. For let $N \in w$. Then there exists $M \in \mathcal{S}$ such that $(M, N) \in \widetilde{B}_{r}$. Hence $N \subset M+B$ $C U S+B=S+B$.

If $E$ is complete, let $B \in Q(\mathrm{UW})$. Then there is a precompact set $S$ such that $S+B \supset \cup w$. Let $S=\{\bar{S}\}$. Since $\bar{S} \in \mathfrak{C}(E)$, it is sufficient to 
prove $W \subset \tilde{B}_{r}(s)$. So let $N \in W$. Then $\bar{S}+B \supset S+B \supset N$, and so $N \in \widetilde{B}_{r}(\delta)$.

Theorem 2. Let $F: T \rightarrow E$ have closed compact values. Among the following statements we have the logical relationship (i) $\Rightarrow(\mathrm{ii}) \Rightarrow(\mathrm{iii})$ :

(i) $F$ is h-condensing;

(ii) $F$ is $r$-condensing;

(iii) $F$ is condensing.

If $E$ is complete, then also (iii) $\Rightarrow$ (ii).

Proof. Let $\Omega \subset T$. By Proposition 3, we have $Q(F(\Omega))=Q\left(\cup F_{h}(\Omega)\right)$ $\supset Q_{r}\left(F_{h}(\Omega)\right) \supset Q_{h}\left(F_{h}(\Omega)\right)$, and $Q(F(\Omega))=Q_{r}\left(F_{h}(\Omega)\right)$ if $E$ is complete. Theorem 2 then follows immediately.

As a consequence of this theorem and Theorem 1 we obtain the following.

Corollary 1. Let $T$ be a nonempty complete convex subset of a separated locally convex space $E$, and let $F: T \rightarrow T$ be a multifunction which has compact convex values, closed graph, and bounded range, and which is either h-condensing or r-condensing. Then $F$ has a fixed point.

4. Lower semicontinuous multifunctions. Recall that a multifunction $F: X \rightarrow Y$ is lower semicontinuous (1.s.c.) iff $F^{-1}(U)$ is open for every open subset $U$ of $Y$. It is well known, and easy to see, that this definition of continuity for a multifunction is different from upper semicontinuity (u.s.c.), which is defined by the requirement that $F^{-1}(B)$ be closed for each closed subset $B$ of $Y$. If $Y$ is regular, $F$ has closed values, and $F$ is u.s.c., then $F$ has closed graph. (See $[5$, p. 175].) Hence the results of $\S \S 2$ and 3 are valid for upper semicontinuous multifunctions. We now turn to the 1.s.c. case.

Lemma 2. Let $X$ be a metric space, and let $F: X \rightarrow X$ be a l.s.c. multifunction with complete values. If there is a nonempty subset $A$ of $X$ such that $\bar{A}$ is compact and $F(A) \subset A$, then there exists a nonempty, closed, and compact set $K$ such that $F(K) \supset K$.

Proof. By Theorem 1.1 of [7] there exists a multifunction $G: X \rightarrow X$ such that $G(x) \subset F(x)$ for all $x \in X, G$ has compact values, and $G$ is u.s.c. As remarked above, $G$ has closed graph. Thus Lemma 2 follows from Lemma 1, since $F(K) \supset G(K)$ for all $K$.

TheOREM 3. Let $T$ be a nonempty complete convex subset of a locally convex space, and let $F: T \rightarrow T$ be a l.s.c. multifunction with closed convex values. Then $F$ has a fixed point if either of the following conditions is satisfied: 
(i) $T$ is compact and metrizable;

(ii) the subspace uniformity on $T$ is metrizable, and $F$ is condensing and has bounded range.

Proof. Assume (i). Then, by Theorem 1.2 of [8], there exists a continuous function $f: T \rightarrow T$ such that $f(x) \in F(x)$ for all $x \in T$. But by Tychonoff's fixed point theorem $f$ has a fixed point, which is also a fixed point of $F$.

Assume (ii). Proceed as in the proof of Theorem 1, until the set $\Omega_{\gamma}$ such that $\Omega_{\gamma+1}=\Omega_{\gamma}$ is obtained. By [5, Corollary 2a, p. 176] we have $F\left(\bar{\Omega}_{\gamma}\right) \subset\left[F\left(\Omega_{\gamma}\right)\right]-\subset \bar{\Omega}_{\gamma}$. Hence apply case (i) to $F \cap\left(\bar{\Omega}_{\gamma} \times \bar{\Omega}_{\gamma}\right): \bar{\Omega}_{\gamma} \rightarrow \bar{\Omega}_{\gamma}$. This is possible since it follows from the inclusion $F\left(\bar{\Omega}_{\gamma}\right) \subset\left[F\left(\Omega_{\gamma}\right)\right]^{-}$ and another application of $\left[5\right.$, Corollary 2a, p. 176] that $F \cap\left(\bar{\Omega}_{\gamma} \times \bar{\Omega}_{\gamma}\right)$ is l.s.c.

Since this manuscript was prepared the authors have become aware of the following related paper: J. Danes, Some fixed point theorems, Comment. Math. Univ. Carolinas 9 (1968), 223-235. Moreover, the referee has pointed out to us the following: E. A. Lifsic and B. N. Sadovskii, $A$ fixed point theorem for generalized condensing operators, Dokl. Akad. Nauk SSSR 183 (1968), 278-279 (Russian).

\section{REFERENCES}

1. C. Berge, Topological spaces, Macmillan, New York, 1963.

2. N. Bourbaki, General topology. Part I, Addison-Wesley, Reading, Mass., 1966.

3. F. E. Browder, On a generalization of the Schauder fixed point theorem, Duke Math. J. 26(1959), 291-303.

4. K. Fan, Fixed-point and minimax theorems in locally convex topological linear spaces, Proc. Nat. Acad. Sci. U.S.A. 38(1952), 121-126.

5. K. Kuratowski, Topology. Vol. I, Academic Press, New York, 1966.

6. E. Michael, Topologies on spaces of subsets, Trans. Amer. Math. Soc. 71(1951), $152-182$.

7. - A theorem on semi-continuous set-valued functions, Duke. Math. J. 26(1959), 647-651.

8. - A selection theorem, Proc. Amer. Math. Soc. 17(1966), 1404-1406.

9. B. N. Sadovskii, A fixed point principle, Functional Anal. Appl. 1(1967), 151153.

10. J. Schauder, Der Fixpunktsatz in Funktionalräumen, Studia Math. 2(1930), 171-180.

11. A. Tychonoff, Ein Fixpunktsatz, Math. Ann. 111(1935), 767-776.

UNIVERSITY OF KANSAS 